\title{
Use of heat resistant V4 Newcastle disease live virus vaccine in rural poultry birds
}

\author{
Ruby Tabassum ${ }^{1}$, Jamila Shafi ${ }^{1}$, Zahid Hussain ${ }^{2 *}$, Javid Iqbal ${ }^{1}$, Sohail \\ Manzoor $^{3}$, Syeda Surriya Gillani ${ }^{4}$, Saima Ali $^{5}$ and Amir Sharif ${ }^{5}$ \\ 1. Livestock and Dairy Development, Poultry Production, Faisalabad-38040-Pakistan \\ 2. Disease Investigations and Control Officer, Livestock and Dairy Development, Bahawalpur Punjab-Pakistan \\ 3. Disease Investigations and Control Officer, Livestock and Dairy Development, Multan Punjab-Pakistan \\ 4. Livestock and Dairy Development, Poultry Production Multan-Pakistan \\ 5. Livestock and Dairy Development, Govt. Poultry Farm Bahawalpur-Pakistan \\ *Corresponding author's email: zhussain60k@gmail.com
}

Citation

Ruby Tabassum, Jamila Shafi, Zahid Hussain, Javid Iqbal, Sohail Manzoor, Syeda Surriya Gillani, Saima Ali and Amir Sharif. Use of heat resistant V4 Newcastle disease live virus vaccine in rural poultry birds. Pure and Applied Biology. Vol. 9, Issue 2, pp1515-1521. http://dx.doi.org/10.19045/bspab.2020.90158

\begin{tabular}{llll}
\hline \hline Received: 03/12/2019 & Revised: 25/02/2020 & Accepted: 06/03/2020 & Online First: 13/03/2020 \\
\hline \hline
\end{tabular}

\section{Abstract}

In this study the comparative immunogenic effect of V4 virus was studied in 700-day old Desi (native) poultry birds through different routes. At the age of $7^{\text {th }}$ day all the birds were divided randomly into seven groups. The birds of Group A to F were given V4 virus vaccine in feed, mixed in wheat flour, Rice, Sorghum, crushed wheat, drinking water and through eye drops respectively. The birds of Group $\mathrm{G}$ were kept as a control group. At the age of 21 days, all the groups were revaccinated with respective vaccines. After primary vaccination, at the age of 21 days, the highest Geometric Mean Titers (GMT 36.99) were noted in-group F and after booster vaccination (age 49 days), highest GMTs value (512) was observed in group F followed by group B (274.37). By 49 days of the age, 10 birds from each group were separated and injected with a virulent Velogenic Newcastle Disease Virus (NDV). The protection percentages were, 50\%, 60\%, $30 \%, 40 \%, 70 \%$ and $80 \%$ in groups A, B, C, D, E and F respectively, while all birds died in group G. The statistically significant differences in Geometric Mean Titers (GMT) Haemagglutination inhibition (HI) antibody titers were found in Group F, than the birds of other groups. The significant differences were also found in HI antibody titers in groups A, B, C, D and E. Results indicated that the $\mathrm{V}-4$ virus vaccine had better protection through ocular drops and in drinking water, followed by in boiled rice-based feed.

Keywords: Heat resistant; ND Vaccine; Rural Poultry; Thermostable; V4 ND Vaccine

\section{Introduction}

In the rural area of Punjab Province of Pakistan, the backyard poultry is still playing a major role to fulfill the nutritional and economic requirements of small land lard agricultural formers. The peoples of rural area of Punjab like to eat meat and eggs from native Desi birds due to their taste and nutritional values and their production on natural sources. In the rural areas of Punjab, the outbreaks of different poultry diseases cause great losses to small and poor farmers. Instead of great efforts by the livestock department, Newcastle Disease is still a big havoc in rural areas, while less prices of commercial poultry meat and eggs as 
compared to rural poultry and better control of disease problems is rising continuously at the rate of $4 \%$ annually in the Pakistan [1]. Recently the federal Government has approved a project "Supply of rural Poultry birds to the farmers" under Prime Minister's Agriculture Emergency Program, amounting to Rs.329.13 million over a period of four years. The salient features of this project are: providing poultry birds and opportunities to the landless farmer, particularly for women, raising a small flock on kitchen and natural waste [1]. This project cannot provide fruitful results in his true spirit, until and unless, the efforts are not, made to control ND outbreaks in rural areas of Pakistan. A recent survey in Punjab province of Pakistan showed that the virulent strains of the NDV (highly pathogenic) are still prevailing in field [2]. To control this disease, the prophylactic vaccination is the only method to control this disease [3]. In Pakistan two types of vaccines (live avirulent and killed oil base) are used in poultry birds, but little work has been done on heat resistant vaccines under rural condition of Desi poultry birds. In the field usually used vaccines are heat sensitive and require proper low temperature facilities from the manufacturer up to the farmers [3, 4].

In this scenario, the heat stable ND vaccine is a good choice for the village level, where it is not possible to maintain the conditions of cold chain supply properly and sometimes even not possible in harsh areas like Cholistan and Thal desserts in Punjab Province. The V-4 ND vaccine is heat resistant strain and is used in drinking water, eye drop, or mixed with feed and this also found good for vaccination of the village Desi poultry [5-7]. The vaccine prepares from V4 strain showed cheering results in the African countries [8]. The V4 strain is a nonvirulent virus and can safely be used in birds of any age from day-old chicks to adult birds $[9,10]$. Keeping in view, this project was started to study and to compare the heat stable vaccine prepare for the V4 virus through different routes.

\section{Materials and methods}

Preparation of ND Vaccine from V4 Strain of virus

A lyophilized V4 strain of ND, Virus was purchased from Veterinary Research Institute (VRI), Lahore. A ten milligrams of lyophilized vaccine material were weighed and was dissolved in $15 \mathrm{ml}$, normal saline having antibiotic (Gentamycine $200 \mu \mathrm{g} / \mathrm{ml}$ ) under sterilized conditions. Twenty, 9 days, fertile chicken eggs were injected with 0.5 $\mathrm{ml}$ freshly prepared viral suspension in each egg by using Chorio-allantoic method rout [11].

After 72 hours of incubation, all the eggs were chilled and Allantoic fluid was sucked with glass pasture-pipette and stored at $20^{\mathrm{O}} \mathrm{C}$ in plastic bottles for future use [12, 13]. Haemagglutination (HA) and Haemagglutination inhibition (HI) method were applied for virus identification and titration of Allantoic fluid [11]. Hyper immune serum against NDV (V4 strain) was raised by repeated injections in rabbits [14, 15]. Fourteen days after the last injection, blood was collected by direct puncture of heart of rabbits and serum was separated. This serum was used for the confirmation of the virus in the vaccine through $\mathrm{HI}$ activity. The lethal dose 50 (LD50) was calculated on chicken embryos in fertilized specific pathogen free (SPF) eggs [16]. To check the contaminations, the Allantoic fluid was directly streaked on Nutrient agar, MacConkey agar, Sabouraud's agar and PPLO broth and incubated at $37^{\circ} \mathrm{C}$ for 48 hours. The Allantoic fluid having V4 virus was directly used as a live vaccine.

\section{Mixing of feed with the V4 Allantoic fluid vaccine}

The Allantoic fluid (V4 virus) was mixed in water having $1 \%$ dry milk powder and further mixed in whole wheat grain flour, 
broken boiled rice; sorghum and compressed wheat for the mixing of V4 vaccine into the feed [6]. The ratio of virus in feed was calculated according to the daily requirement of feed and the required dose of LD 50. Other methods of vaccine delivery were in drinking water and eye drops.

\section{Experimental trial}

A total 700-day old village poultry birds were purchased from a local hatchery and they were kept in a village of District Toba Tek Singh. At the age of $7^{\text {th }}$ day, birds were divided into 7 groups (100 birds in each) and vaccinated as below.

Group A: V4 Virus in whole wheat flourbased vaccine.

Group B: V4 Virus in boiled Rice-based vaccine.

Group C: V4 Virus mixed in sorghumbased vaccine.

Group D: V4 Crushed wheat-based vaccine.

Group E: V4 in drinking water.

Group F: V4 Eye drop vaccine

Group G: Kept as Control.

At the age of 21days, these groups were revaccinated with the same vaccine as mention above.

\section{Assessment of vaccines}

The effectiveness of the trial vaccine was checked on the values of HI antibody titers at days 7, 14 and 21 after primary vaccination and at 28, 35, 42 and 49 days after post booster vaccination [11]. Ten birds, from each group at the age of 49 days were infected with a Viscerotropic Velogenic Newcastle Disease (VVND) virus at a rate of $0.1 \mathrm{ml}$ virus intramuscularly per birds. The typical lesions were recorded carefully for 10 days after challenge infection.

\section{Statistical analysis}

Data was accessed by Geometric mean titers and through analysis of means applying randomized complete design [17].

\section{Results and discussion Humoral immune response}

Ranikhet or Newcastle Disease (ND) is still considered internationally as a one of the important diseases of chicken and other poultry birds, due to its bad consequences in infected birds, which increase the mortality reaching up to $100 \%$ [8]. This disease cannot be cured by medicines; the only way of controlling ND is through an effective vaccination program. In many countries feed-based vaccines are developed mainly to save village/rural birds from this disease [6]. But there are some basic problems, which are still present with the vaccination of village poultry birds. The present study was conducted to solve these problems and to get rid of expensive cold chain facilitation in harsh and hot weather. During this study immune response in each experimental group was accessed both after primary and booster vaccination by HI GMT antibody titers (Fig. 1).

Results showed that, at the start of the experimental (day zero), GMTs were 7.60, 7.40, 2.60, 5.20, 6.60, 6.80 and 5.80 in groups A, B, C, D, E, F and G respectively. On day $7^{\text {th }}$, after primary vaccination, a significant $(p \leq 0.01)$ Difference was noted between all the groups. Similarly, on day $14^{\text {th }}$ (age 21) of post-vaccination, significant differences between GMTs of all groups were recorded. The same trend was also recorded in all groups after post booster vaccination at the age of 28, 35, 42 and 49 days. The statistical data analysis provided significant difference between all the groups, but the group F had highly significantly $(\mathrm{p} \leq 0$. 01) GMT titers among all the groups (Fig. 2)

Results showed that groups $\mathrm{F}$ and $\mathrm{E}$ had better immune response on day 49 of the experiment; however, the results of V4 vaccine given in boiling rice are also encouraging. The statistical analyses of the results are presented in (Table 1). 


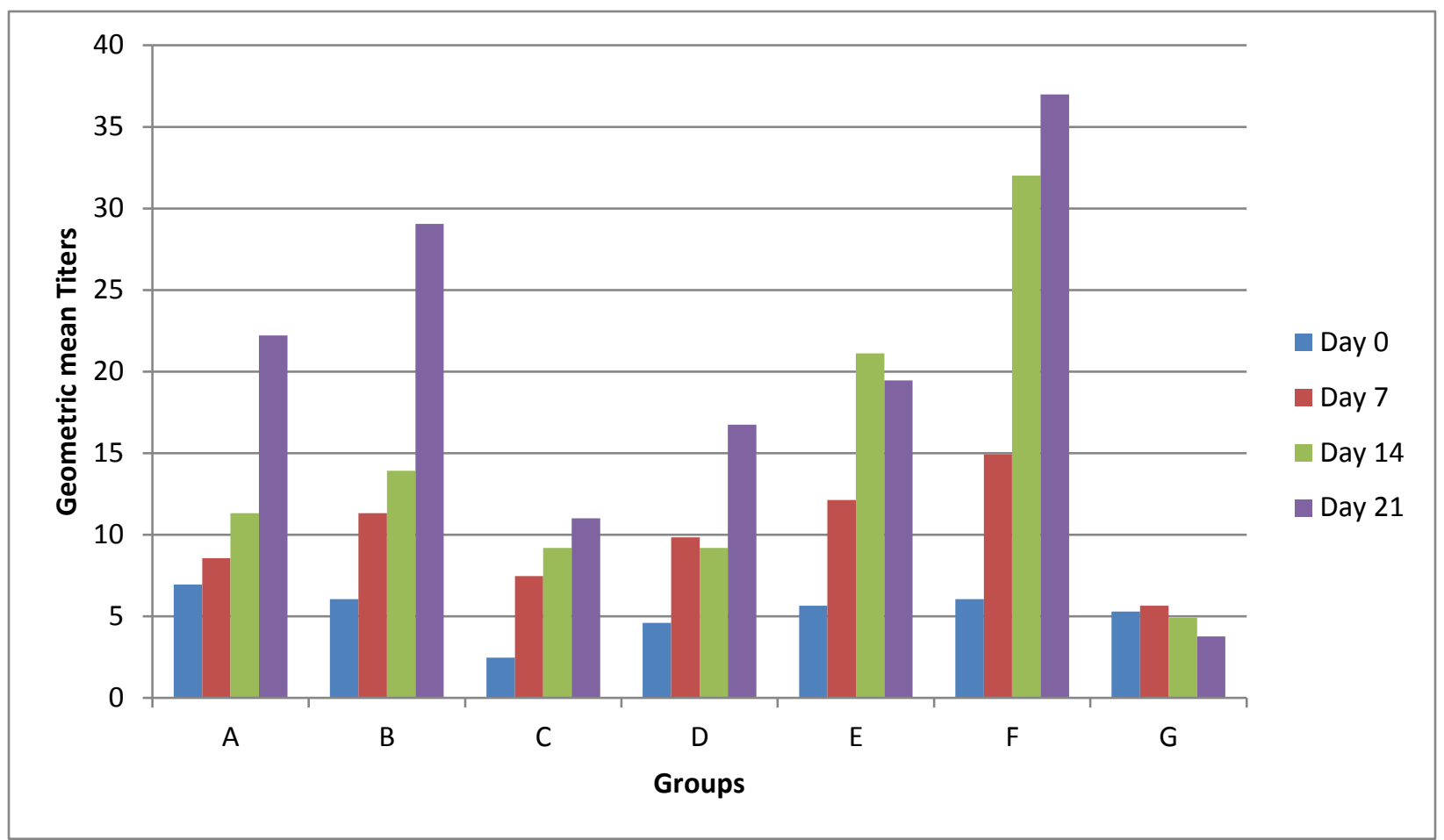

Figure 1. GMT. HI. Antibody titers in groups after primary vaccination

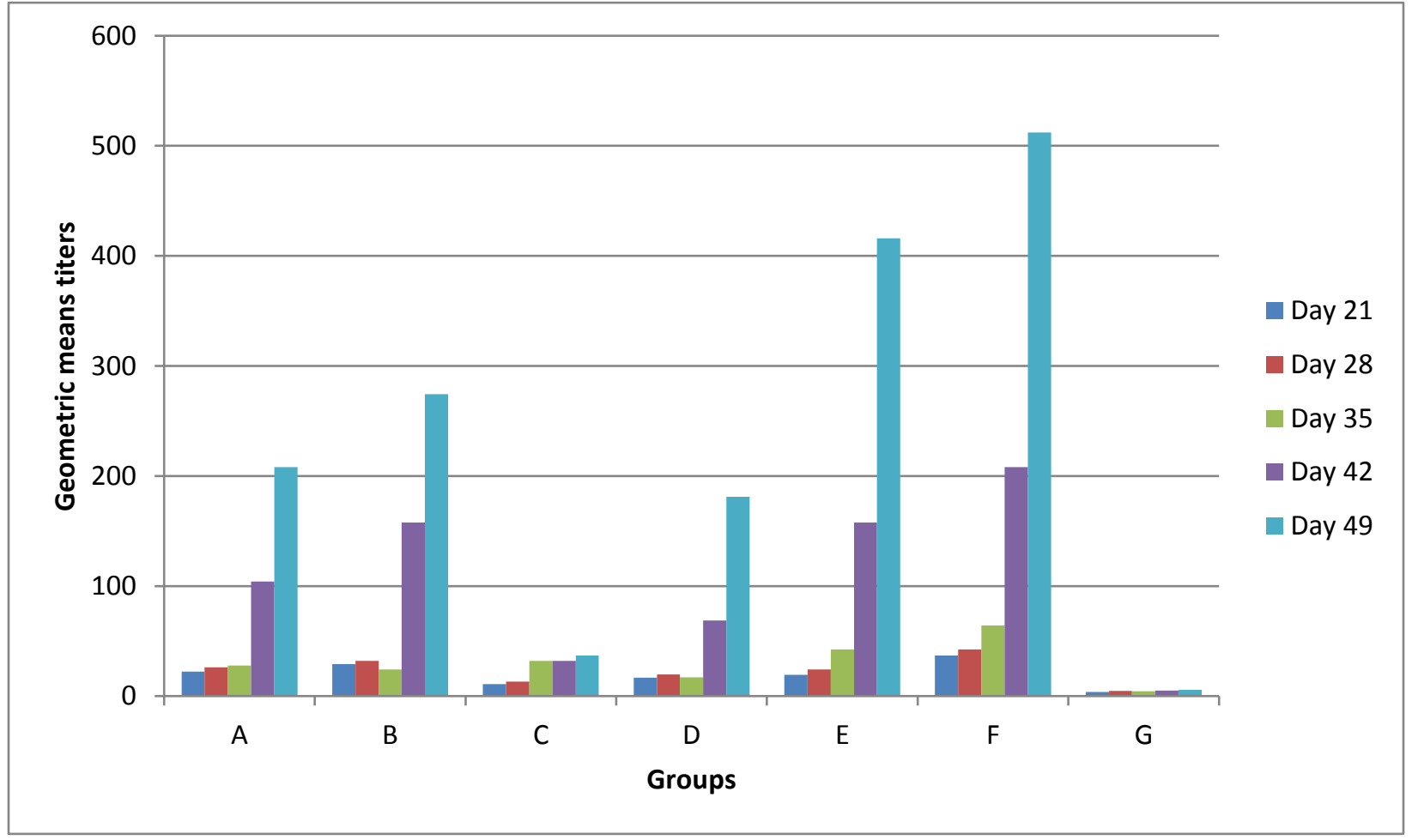

Figure 2. Immune response after booster vaccination 
Table 1. Mean \pm SE Post-vaccination and post-booster GMT $(n=10)$ of different experimental groups

\begin{tabular}{|c|c|c|c|c|c|c|c|}
\hline \multirow{3}{*}{ Groups } & \multicolumn{3}{|c|}{$\begin{array}{c}\text { GMT means after Primary } \\
\text { Vaccination }\end{array}$} & \multicolumn{3}{c|}{ GMT Means after Booster vaccination } \\
\cline { 2 - 8 } & \multicolumn{3}{|c|}{ Age in days } & \multicolumn{3}{c|}{ Age in days } \\
\cline { 2 - 8 } & $\mathbf{7}$ & $\mathbf{1 4}$ & $\mathbf{2 1}$ & $\mathbf{2 8}$ & $\mathbf{3 5}$ & $\mathbf{4 2}$ & $\mathbf{4 9}$ \\
\hline \multirow{2}{*}{$\mathbf{A}$} & $7.60^{*}$ & $10.00^{* *}$ & $13.20^{* *}$ & $28.80^{* *}$ & $30.40^{* *}$ & $124.80^{* *}$ & $243.20^{* *}$ \\
& \pm 1.11 & \pm 1.71 & \pm 2.53 & \pm 4.65 & \pm 4.30 & \pm 24.53 & \pm 48.46 \\
\hline \multirow{2}{*}{$\mathbf{B}$} & $7.40^{*}$ & $13.60^{* *}$ & $16.00^{* *}$ & $36.80^{* *}$ & $30.40^{* *}$ & $172.80^{* *}$ & $320.00^{* *}$ \\
& \pm 1.58 & \pm 2.61 & \pm 2.92 & \pm 6.33 & \pm 6.40 & \pm 23.47 & \pm 54.81 \\
\hline \multirow{2}{*}{$\mathbf{C}$} & $2.60^{*}$ & $8.00^{* *}$ & $10.80^{* *}$ & $13.60^{* *}$ & $35.20^{* *}$ & $36.80^{* *}$ & $41.60^{* *}$ \\
& \pm 0.31 & \pm 1.03 & \pm 1.79 & \pm 1.22 & \pm 5.23 & \pm 6.33 & \pm 6.40 \\
\hline \multirow{2}{*}{$\mathbf{D}$} & $5.20^{*}$ & $10.80^{* *}$ & $10.00^{* *}$ & $24.00^{* *}$ & $20.00^{* *}$ & $76.80^{* *}$ & $230.40^{* *}$ \\
& \pm 0.80 & \pm 1.47 & \pm 1.37 & \pm 5.33 & \pm 3.43 & \pm 11.88 & \pm 52.43 \\
\hline \multirow{2}{*}{ E } & $6.60^{*}$ & $13.60^{* *}$ & $22.40^{* *}$ & $30.40^{* *}$ & $44.80^{* *}$ & $192.00^{* *}$ & $460.80^{* *}$ \\
& \pm 1.27 & \pm 2.40 & \pm 2.61 & \pm 7.33 & \pm 5.23 & \pm 42.67 & \pm 74.39 \\
\hline \multirow{2}{*}{ F } & $6.80^{*}$ & $17.60^{* *}$ & $40.00^{* *}$ & $44.80^{* *}$ & $73.60^{* *}$ & $243.20^{* *}$ & $588.80^{* *}$ \\
& \pm 1.20 & \pm 3.33 & \pm 8.00 & \pm 5.23 & \pm 12.67 & \pm 48.46 & \pm 101.33 \\
\hline \multirow{2}{*}{ G } & $5.80^{*}$ & $6.20^{* *}$ & $5.60^{* *}$ & $5.20^{* *}$ & $5.20^{* *}$ & $5.20^{* *}$ & $6.60^{* *}$ \\
& \pm 0.78 & \pm 0.76 & \pm 0.83 & \pm 0.80 & \pm 0.80 & \pm 0.80 & \pm 1.27 \\
\hline
\end{tabular}

*Significant, **Highly Significant $(\mathrm{P} \leq 0.01)$

Highest antibody titers were observed in groups vaccinated through eye drops and in drinking water, followed by rice-based vaccine. Using the eye drops method ensures that each chicken receives a sufficient dose of vaccine in close proximity of the Harderian gland, an immune organ in a chicken eye. The Harderian gland in birds produces antibodies and helps inform the development of the immunity in birds. With ocular vaccination a handsome amount of the vaccine virus enters into the Harderian gland and a quick immune response is started [6]. These findings were also in agreement with the works of other workers [18]. The eye-drop method is thus better recommended for ND vaccination in chickens [8]. Mixed poultry stocks are kept in the rural area, poultry management systems and even chickens of different age groups, sizes and in some cases different poultry species are raised together. It was noted that rural chickens scrambled during day time and vaccination through drinking water became a disadvantage for the chicks and other weaker birds. It is therefore possible that the birds did not take an adequate vaccine in the drinking water. These factors result in the low immunity development in birds given the vaccine orally. To solve these difficulties the vaccines, which, can be delivered in feed are developed for village birds against NDV. This has been encouraged by the difficulty of catching spread village chickens for routine vaccination [6]. Higher antibody titer with eye drops and drinking water were achieved followed by parboiled rice vaccine $[7,19]$. In village boiled rice is a common food consumed by the peoples and it has also been used for chicken feed in the form of home scraps, but the problem with this is that, it rapidly putrefied with contamination [6]. In this study boiled rice also did not show as a good carrier for V4 ND vaccination. In other countries like Malaysia, the crushed wheat as a vaccine carrier had also showed encouraging results during trials, where it is mixed with feed in large feed mixers and stored for some weeks at room temperature [6]. The lowest antibody titers were observed 
with sorghum. These results are also supported by a previous study conducted in Ethiopia with the heat resistant NDV-12 vaccine against challenge with a virulent velogenic stain of NDV [20].

\section{Challenge protection trial}

In challenge protection trail, total mortality was recorded in 10 days, Group F which was administered experimental vaccine through eye drops, showed a maximum (80\%) protection while $100 \%$ mortality was recorded in the control group. The results of challenge protection trial are given in (Fig.
3). The $50 \%$ chicken remained alive vaccinated with vaccine in crushed wheat, $60 \%$ in boiling rice-based vaccine, $30 \%$ in sorghum-based vaccine, $40 \%$ via wheat flour vaccine, $70 \%$ in drinking water respectively, while all birds died in group $\mathrm{G}$. The vaccine prepared from V4 stain provides good results via ocular method. Previous study also showed that V4 ND, Virus at a temperature of $25-28^{0} \mathrm{C}$ is thermostable and retain its effectivity for many weeks at $28^{0} \mathrm{C}$ environmental temperatures in freeze-dried form [21].

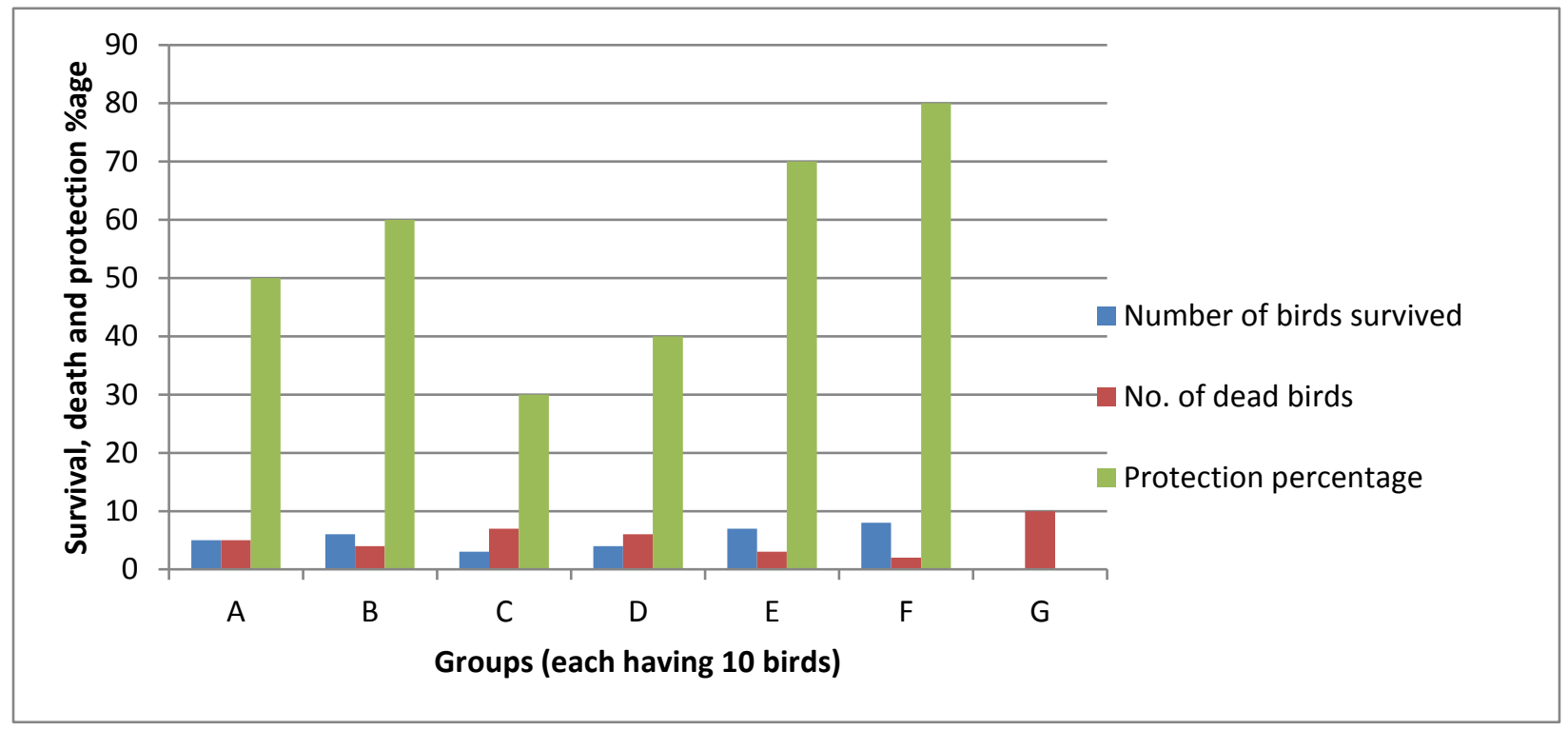

Figure 3. Mortality percentage with challenge protection Test

\section{Conclusion}

This study proved that, a V-4 ND live vaccine is effective and immunogenic against local ND strains.

\section{Authors' contributions}

Conceived and designed the experiments: $\mathrm{R}$ Tabassum, Performed the experiments: J Shafi, SS Gillani \& S Ali, Analyzed the data: $\mathrm{Z}$ Hussain \& S Manzoor, Contributed reagents/materials/analysis tools: J Iqbal, $S$ Ali \& A Sharif, Wrote the paper: Z Hussain.

\section{References}

1. Pakistan Economic Survey (2018-19). Chap. 2. Agriculture, pp 14.
2. Annual Disease report (2018). Poultry Research Institute Rawalpindi.

3. Usman M (2002). Effects of vaccination of chickens against Newcastle disease with thermostable $\mathrm{V}_{4}$ and LaSota vaccines using different grains and their brand as a vehicle. MSc Thesis, Department of Veterinary Surgery and Medicine, Ahmadu Bello University, Zaria, Nigeria.

4. Anne I, Ibrahim AL \& Spradbrow PB (1990). Field trials of a food-based vaccine to protect chickens against 
Newcastle disease. Res Vet Sci 49: 216219.

5. Saglid IK \& Spalatin J (1982). Newcastle disease Vaccination with the $\mathrm{V}_{4}$ strain in Malawi: Laboratory and field studies. Avian Dis 26: 625-628.

6. Spradbrow PB (1992). A review of the use of food carriers for the delivery of oral Newcastle disease vaccine. In: Proceedings of an International Workshop, Kuala Lumpur, 6-10 October, Canberra, ACIAR Proceedings 39: 18-20.

7. Tu T, Phuc KV, Dinh NTK, Quoc DN \& Spradbrow PB (1998). Vietnamese trials with a thermostable Newcastle disease vaccine (strain 9-2) in experimental and village chickens. Prevent Vet MED 34: 205-214.

8. Alders R \& Spradbrow PB (2001). Controlling Newcastle disease in village chickens. A Field Manual. ACIAR, Canberra, Australia. pp 1-70.

9. Spradbrow PB (1993). Newcastle disease in Village Chickens. Poult Sci Rev 5: 57-96.

10. Spradbrow PB (1994). Newcastle disease in village chickens. Poult Sci Rev 39: 18-2.

11. OIE (2009). Manual of Diagnostic Tests and Vaccines for Terrestrial Animals: Newcastle Dis 576-589.

12. Mughal UFR (2002). Efficacy of live oil adjuvanted LaSota strain Newcastle disease vaccine in boiler chicks under experimental conditions. M.Sc. Thesis. Deptt. Vety Micro, Univ Agri, Fsd, Pakistan

13. Malik BS (2003). A laboratory manual of Veterinary Microbiology. Part-IV (Virology) CBS Publishers and Distributors. New Dehli, India.
14. Manzoor S \&. Hussain I (2003). Reverse Passive Haemagglutination Assay (RPHA) test for detection and quantification of Hydro pericardium Syndrome Virus (HPSV). Polk, J Life Sci 1 (2): 141-43.

15. Islam MS, Parvin MS, Akhtar J, Islam MT, Sidiqque MP \& Rashid MH (2013) Clinical Evaluation of Hyperimmune Serum for the Treatment of Newcastle Disease in Indigenous Layer Birds. Progress Agric 24 (1 \& 2): 79-84.

16. Reed LJ \& Muench H (1938). A simple method of estimating fifty percent endpoints. American J Hygiene 27: 493497.

17. Steel RGD, Torrie JH \& Dickey DA (1997). Principles and Procedures of Statistics. A biometrical approach (3rd Ed), McGraw Hill Book Co., New York, USA.

18. Wambura PN, Kapaga AM \& Hyera JMK (2000). Experimental trials with a thermostable Newcastle disease virus (strain I2) in commercial and village chickens in Tanzania. Prev Vet MED 43: 75-83.

19. Samuel JL \& Spradbrow PB (1991). Selective oral vaccination against Newcastle disease by creep feeding young chicks in an open range poultry flock. Prev Vet Med 10: 273-282.

20. Nasser M, Lohr JE, Mebratu GY, Zessin KH, Baumann MPO \& Ademe Z (2000). Oral Newcastle disease vaccination trials in Ethiopia. Avian Path 29: 27-34.

21. Ideris A1, Ibrahim A L\& Spradbrow P B (1990). Vaccination of chickens against Newcastle disease with a food pellet vaccine. Avian Path 19(2): 371-84. 